\title{
LOCALITIES AND SITES OF THE SOUTHEASTERN-ALPINE ENDEMIC SPECIES SPIRAEA DECUMBENS KOCH IN BREGINJSKI KOT (NORTHWESTERN SLOVENIA) NAHAJALIŠČA IN RASTIŠČA JUGOVZHODNOALPSKEGA ENDEMITA SPIRAEA DECUMBENS KOCH V BREGINJSKEM KOTU (SEVEROZAHODNA SLOVENIJA)
}

\author{
Marko PAVLIN ${ }^{1}$, Robert BRUS ${ }^{2}$, Igor DAKSKOBLER ${ }^{2,3}$ \\ (1) Borjana, 20, SI-5223 Breginj, markopavlin4@gmail.com \\ (2) University in Ljubljana, Biotechnical Faculty, Department of Forestry and Renewable Forest Resources, Večna pot 83, SI-1000 \\ Ljubljana, robert.brus@bf.uni-lj.si \\ (3) Institute of Biology, Scientific Research Centre of the Slovenian Academy of Sciences and Arts, Regional Unit Tolmin, Brunov \\ drevored 13, SI-5220 Tolmin, igor.dakskobler@zrc-sazu.si
}

\begin{abstract}
The eastern distribution limit of Spiraea decumbens s. str. is in Breginjski Kot (northwestern Slovenia), where we recorded 49 localities of this eastern-Alpine endemic plant species. We determined two new localities in a new quadrant (9746/4) on riparian rocks of the Nadiža River under Mt. Mija at Kozja Peč, which is currently the southeasternmost point of its entire distribution area. We conducted a phytosociological survey of 26 localities. This endemic occurs in dolomite rock crevices in stands classified within the association Spiraeo-Potentilletum caulescentis. On dolomite screes and torrential fens it is found to dominate on small areas in the successional stages, classified into the provisional association Aquilegio einseleanea-Spiraeetum decumbentis (alliance Petasition paradoxi) and into the new association Spiraeo decumbentis-Seslerietum calcariae (alliance Caricion austroalpinae). These stages are gradually becoming overgrown with thermophilous deciduous trees, and in one of the relevés we found the studied species in an open hop hornbeam stand classified into the association Fraxino orni-Ostryetum. Its populations are vital and unthreatened, except in the two new localities under Mt. Mija, which call for special protection due to their small size and a possibility of being backfilled with gravel.
\end{abstract}

Key words: phytogeography, phytosociology, population ecology, Spiraea decumbens subsp. decumbens,

Breginjski Kot, Slovenia

\begin{abstract}
IZVLEČEK
V Breginjskem kotu (severozahodna Slovenija) smo popisali 49 nahajališč vzhodnoalpskega endemita polegle medvejke (Spiraea decumbens s. str.), ki ima tu vzhodno mejo svojega areala. Ugotovili smo dve novi nahajališči v novem kvadrantu (9746/4), in sicer na obrečnih skalah Nadiže pod goro Mijo pri Kozji peči, kar je za zdaj skrajna jugovzhodna točka celotnega areala. 26 nahajališč smo fitocenološko popisali. Sestoje, kjer ta endemit uspeva v dolomitnih skalnih razpokah, uvrščamo v asociacijo Spiraeo-Potentilletum caulescentis. Na dolomitnih meliščih in hudourniških vršajih je na manjših površinah prevladujoč v sukcesijskih stadijih, ki jih uvrščamo v provizorno asociacijo Aquilegio einseleanea-Spiraeetum decumbentis (zveza Petasition paradoxi) in v novo asociacijo Spiraeo decumbentis-Seslerietum calcariae (zveza Caricion austroalpinae). Ti stadiji se postopno zaraščajo s toploljubnimi listavci in na enem popisu smo preučeno vrsto našli v vrzelastem sestoju črnega gabra, ki ga uvrščamo v asociacijo Fraxino orni-Ostryetum. Njene populacije so vitalne in neogrožene, razen na obeh novih nahajališčih pod Mijo, ki bi zaradi majhnosti in mogočega zasutja s prodom morali biti deležni posebne varstvene pozornosti.
\end{abstract}

Ključne besede: fitogeografija, fitocenologija, populacijska ekologija, Spiraea decumbens subsp. decumbens, Breginjski kot, Slovenija

GDK= 181.1:176.1Spiraea decumbens s. str.(497.4Breginjski kot)(045)=111 Prispelo / Received: 27.03. 2015 Sprejeto / Accepted: 28. 10. 2015

\section{INTRODUCTION}

1 UVOD

Spiraea decumbens is a southeastern-Alpine endemic plant species distributed in northeastern Italy and northwestern Slovenia. We distinguish two subspecies, $S$. decumbens Koch subsp. decumbens, which occurs in northwestern Slovenia and northeastern Italy, and $S$. decumbens subsp. tomentosa (Poech.) Dostal. = S. decumbens subsp. hacquetii (Fenzl \& Koch) Arcang., which occurs in the eastern part of its distribution area, only in northeastern Italy (Aeschimann et al., 2004; Poldini,
2002). The distribution of this endemic plant species in Slovenia was discussed by Wraber (1969), Wraber and Skoberne (1989), Jogan (2000), Dakskobler (2003) and Čušin (2006). They reported the following localities: Tam v Klinu, Tam na Lepem Brdu under Mt. Mali Muzec (Musc), along the Bela above Breginj, at Most na Nadiži (Ponte Vittorio) and under Drnohla above the Učja valley. All its localities known so far in Slovenia have been under the Stol ridge above the Učja valley and in Breginjski Kot. Spiraea decumbens is listed as rare in the Red List (Anon., 2002). So far, we have conducted 
phytosociological investigations only of the sites in the rock crevices above the Učja valley, at Plazi and under the mountain pasture Ohoje in Breginjski Kot. These relevés were temporarily assigned to the association Potentilletum caulescentis s. lat. (Dakskobler, 2003). The purpose of our study was to record as many localities of this rare endemic species in Breginjski Kot as possible, to study the plant communities on its sites and assess its vitality and potential threat.

\subsection{Ecological description of the study area}

1.1 Ekološki opis raziskovanega območja

Breginjski Kot is situated at the foothills of the Julian Alps and is the westernmost part of Slovenia. It is surrounded by the Stol Mountains in the north, the Nadiža River in the west, by mounts Mija and Ljubija in the south and extends into Staroselsko Podolje lowland in the east. The region has a moist climate with mean annual precipitation exceeding $2500 \mathrm{~mm}$ and periodically heavy rain. The bedrock at higher elevations is composed of hard calcareous rocks, dolomite, dolomitised limestone and limestone, while the sub-montane belt is dominated by flysch and glacial deposits. The vegetation reflects strong anthropozoogenous influence. Steep and stony areas that were unsuitable for farming are overgrown with forest. Other areas are mainly covered by grasslands that have been subjected to rapid overgrowing in recent decades. The composition of the local flora is dominated by plants of the moderate climatic belt and plants that belong to the boreal geoelement. There are many Mediterranean-montane, Mediterranean and Illyrian species, of which 23 are endemic, mainly southeastern-Alpine (Čušin, 2006).

\section{MATERIALS AND METHODS}

\section{MATERIALI IN METODE}

In Breginjski Kot, we examined the areas with already known localities and the areas where this species could potentially occur. We used the GPS receiver and a synoptic map where we marked the localities. The coordinates of the localities were entered into the FloVegSi database (Seliškar et al., 2003). For a more detailed analysis we selected six areas where we made 26 relevés, applying the standard Central-European method (Braun-Blanquet, 1964). These relevés were entered into the FloVegSi database. Combined cover-abundance values were transformed into ordinal values 1 - 9 (van der Maarel, 1979). Numerical comparisons were made with the software package SYN-TAX 2000 (Podani, 2001). Relevés were arranged into the analytic table (Table 1) based on hierarchical classification and assessment of diagnostic species. We integrated the results of the (unweighted) pair group method with arithmetic mean "(Unweighted) average linkage" - UPGMA, where Wishart's similarity ratio was applied. Phytosociological groups (= groups of diagnostic species) were formed on the basis of our own criteria, but with consideration of numerous authors. The nomenclature source for the names of vascular plants is Martinčič et al. (2007), and Martinčič $(2003,2011)$ for the names of mosses. The nomenclature source for the names of syntaxa is Šilc and Čarni (2012). On each locality we determined the size of the populations and assessed their vitality and potential threat. Vitality was scored on a scale where 1 = poor, 2 = moderate and 3 $=$ good.

\section{RESULTS AND DISCUSSION}

\section{REZULTATI IN RAZPRAVA}

\subsection{Phytosociological description of the sites of} Spiraea decumbens in Breginjski Kot

3.1 Fitocenološka oznaka rastišč vrste Spiraea decumbens $\mathrm{v}$ Breginjskem kotu

The 26 relevés in Table 1 were arranged based on hierarchical classification and with consideration of diagnostic species. We found that the relevés in rock crevices and ledges are floristically very similar to those on steep gravel directly under rock faces, as chasmophytic species frequently occur also on screes, and vice versa. Spiraea decumbens usually spreads abundantly on screes and its coverage is considerably higher there than in rock crevices. Relevés 1 and 2 in Table 1 that were made in the area of Kozja Peč are unique and cannot be adequately syntaxonomically classified as yet, but they belong to a chasmophytic community (class Asplenieta trichomanis). Relevés 2 to 10 in Table 1 can be classified into the association Spiraeo decumbentis-Potentilletum caulescentis Poldini 1969. Compared with the stands of this association in the Carnic Alps and in the Italian part of the Julian Alps (Poldini, 1973) they are somewhat floristically impoverished. Some of the species, for example Leontodon tenuiflorus, Athamanta turbith, Primula auricula, which are common or diagnostic in the original table of this association, were therefore not recorded on our plots. Our stands are treated as a special variant with Rhodothamnus chamaecistus, which very rarely occurs in relevés from Italy. The differential species of the variant is also Campanula cespitosa. Relevés 12 to 16 in Table 1 characterise small ledges or steep screes under rock faces, i.e. scree vegetation, which is in fact usually dominated by Spiraea decumbens. For the time being, these stands are named after Spiraea decumbens and Aquilegia einseleana as the provisional associati- 
on Aquilegio einseleanae-Spiraeetum decumbentis nom. prov. Its diagnostic species are Spiraea decumbens and Aquilegia einseleana, and its differential species are Salix glabra, Campanula cespitosa, Leontodon hispidus subsp. hyoseroides and Rhamnus pumilus. This is a successional stage that occurs in small areas (from $2 \mathrm{~m}^{2}$ to $10 \mathrm{~m}^{2}$ ) on initial soil (lithosol) in the overgrowing of dolomite gravel or of rock ledges. It is characterised by coexistence of chasmophytic species, scree species and species of stony subalpine grasslands. This stage, which requires further investigation, is temporarily classified into the alliance Petasition paradoxi, order Arabido alpinae-Petasitetalia paradoxi and class Thlaspietea rotundifolii. Relevés 17 to 25 in Table 1 represent the next stage in succession, extensively overgrown established screes or gravelly grasslands in erosion areas that are very slowly becoming overgrown with shrub and tree species. These stands are classified into the new association Spiraeo decumbentis-Seslerieteum calcariae ass. nov. Its diagnostic species are Spiraea decumbens, Sesleria caerulea subsp. calcaria, Erica carnea, Polygala chamaebuxus, Molinia caerulea subsp. arundinacea, Allium ericetorum, Polygala nicaeensis subsp. carniolica (= P. nicaeensis subsp. forojulensis) and Chamaecytisus purpureus. The nomenclature type of the new association, holotypus, is relevé 21 in Table 1. The new association is classified into the alliance $\mathrm{Ca}$ ricion austroalpinae, order Seslerietalia coeruleae and class Elyno-Seslerietea. In the next stage of succession, these overgrown screes are sometimes covered with open Ostrya carpinifolia and Fraxinus ornus forest classified into the association Fraxino orni-Ostryetum. An example of such an open forest can be seen in relevé 26 in Table 1.

Synsystematic classification of the studied communities into higher units is as follows:

Class: Asplenieta trichomanis (Br.-Bl. in Meier \& Br. Bl. 1934) Oberd. 1977

Order: Potentilletalia caulescentis Br.-Bl. in Br.-Bl. et Jenny 1926

Alliance: Phyteumato-Saxifragion petraeae Mucina in Mucina et al. 2011

Association: Spiraeo decumbentis-Potentilletum caulescentis Poldini 1969

var. Rhodothamnus chamaecistus var. nov.

Class: Thlaspietea rotundifolii Br.-Bl. 1948

Order: Arabido alpinae-Petasitetalia paradoxi $\mathrm{Mu}$ cina et al. 2012

Alliance: Petasition paradoxi Zollitsch ex Lippert 1966

Association: Aquilegio einseleanae-Spiraeetum decumbentis nom. prov.

Class: Elyno-Seslerietea Br.-Bl. 1948

Order: Seslerietalia caeruleae Br.-Bl. et Jenny 1926 Alliance: Caricion austroalpinae Sutter 1962

Association: Spiraeo decumbentis-Seslerietum calcariae Dakskobler in Pavlin,

Brus et Dakskobler 2015 ass. nov.

Class: Querco-Fagetea Br.-Bl. et Vlieger in Vlieger 1937

Order: Quercetalia pubescenti-petraeae Klika 1933 Alliance: Fraxino orni-Ostryion Tomažič 1940 Association: Fraxino orni-Ostryetum Aichinger 1933 corr. Franz 2002

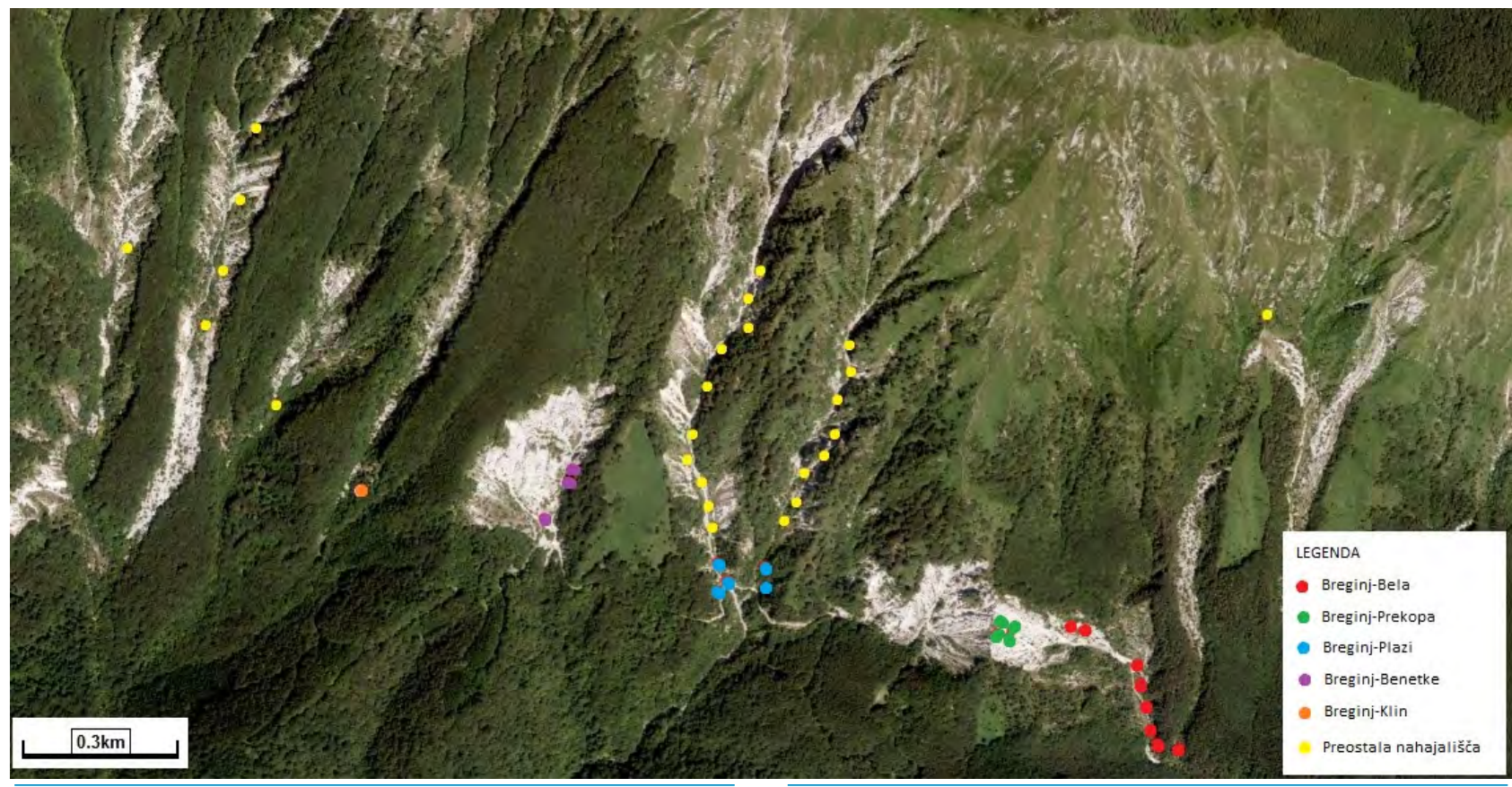

Fig. 1: Localities of Spiraea decumbens under the Stol ridge

Slika 1: Nahajališča vrste Spiraea decumbens pod Stolovim grebenom 


\begin{tabular}{|c|c|c|c|c|c|c|c|c|c|c|c|c|c|c|c|c|c|c|c|c|c|c|c|c|c|c|}
\hline & & & & & & & & & & & & & & & & & 立产 & pim & $\bar{m}$ & $\hat{N}$ & $\simeq$ & $\infty 0$ & & $\nabla$ & $\nabla \sigma$ & $\nabla+$ \\
\hline & & & & & & & & & & & & & & & & & $\dot{a} \cong$ & ㄴ) & $\infty$ & & & & & - & $-r$ & -- \\
\hline & 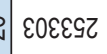 & ని & 3 & ก) & ভ̄ & & 의 & 2. & $\infty$ & চ & పָ & $\forall 10 Z / / Z / 9$ & u!!|પ્ર-[u!̣әرg & $l / 9 t<6$ & $Z 669 \angle \varepsilon$ & $\varepsilon \angle E L Z L G$ & $m$ & $m$ & & & & & & & & \\
\hline & เ6ZE९乙 & శి & ш & \& & (ড) & $\Xi$ & 8 & L & 8 & ন & $\sqrt[4]{4}$ & $\nabla L O Z / L Z / S$ & edoyәдd-[u!!దəдg & $2 / 9+\angle 6$ & Z৪Z8LE & 990LZLG & $r$ & - & - & + & & & & & & \\
\hline & †6乙๕ऽ乙 & প্র & 3 & 8 & ○: & $\Xi$ & 8 & & $\infty$ & $\mid 5$ & $2 m$ & $\nabla L O Z / L Z / S$ & 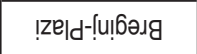 & $b / 9 t<6$ & $\angle G L L L E$ & GZLLZІG & - & - & + & & & & & & & \\
\hline & 8LZESZ & 8 & $\infty$ & $\infty$ & ভ̄ & $\propto$ & ما & & ஜ & ले & $n$ & $\nabla 10 Z / L Z / 9$ & е|әg-โu!̣ڤəגg & $Z / 9+\angle 6$ & $L \angle S 8 L E$ & 9989ZlG & $m$ & $m$ & & & & & & & & \\
\hline & 89z๕૬乙 & 요 & क & $\infty$ & ঠे & $\simeq$ & 은 & & ৪ & $F$ & $=\mathrm{m}$ & $t \downarrow L Z / L Z / s$ & е|әg-โu!̣ڤəגg & $Z / 9 t \angle 6$ & L LS8LE & 9989ZlG & $m$ & $m$ & & & & & & & & \\
\hline & 18Z\&९Z & প & $\infty$ & $\infty$ & ঠे & & ৪্ল & & P & 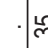 & :̊̊ & $\forall 10 Z / L Z / G$ & 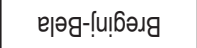 & $Z / 9+\angle 6$ & $8 \varepsilon 98 \angle \mathcal{E}$ & 9969ZlS & $m$ & $m$ & & & & & & & & \\
\hline & 08Z\&९乙 & $\frac{1}{2}$ & $\infty$ & $\infty$ & ড) & $\simeq$ & ㄱ. & & $\infty$ & ล & 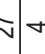 & tレOZ/LZ/S & е|әg-[u!̣ڤəдg & $Z / 9 t \angle 6$ & LLS8LE & 9989ZlG & r & v & & & & & & & & \\
\hline & ๑6乙๕५乙 & প & ш & 아 & ভे: & ت & ০ & & P & in & $=\sqrt{-}$ & $\nabla L O Z / L Z / G$ & 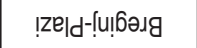 & $L / 9 t<6$ & 6LLLLE & OELLZLS & $\theta$ & o & & & & & & & & + \\
\hline & LLZ६९乙 & : & फ & $\stackrel{2}{\leftarrow}$ & ঠे & & م & & \& & $\stackrel{m}{\sigma}$ & $=\sim 2$ & i & 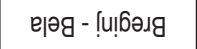 & $2 / 9+\angle 6$ & 乙૬९८LE & 9L89ZLG & $m$ & m & & & & & & & & \\
\hline & L9ZESZ & |ூి & $\omega$ & 으 & 0 & $\simeq$ & s & & 웅 & ले & 吕은 & $\nabla\llcorner O Z / L Z / S$ & 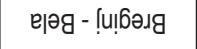 & $2 / 9+\angle 6$ & $0998 \angle \varepsilon$ & 9989ZLG & m & m & & & & & & & & \\
\hline & 66Z६९乙 & న్రి & $z$ & 우 & 0 & $\propto$ & o & r & $\infty$ & & $\therefore \sim$ & $\neq 10 Z / L Z / G$ & 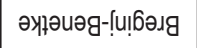 & $1 / 9+\angle 6$ & ZSELLE & 08ZLZLG & $\nabla$ & o & & + & & & & & & \\
\hline & LOE\&GZ & 怘 & ळ & 우 & 口: & $\Xi$ & 아 & & 8 & $F$ & $=\left[\begin{array}{ll}n \\
m\end{array}\right.$ & 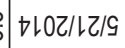 & әуґәиәg-!u!దәдg & $l / 9 t<6$ & ルサLLE & $66 \varepsilon \angle Z L 9$ & $\nabla$ & A & + & & & . & & & & \\
\hline & $\downarrow \nabla 8 Z \varepsilon \hookrightarrow 乙$ & ఎి & ш & m & ভ̄: & $二$ & ল & శొ & is & 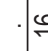 & $=$ & $=\nabla 10 Z / L Z / S$ & е|әg-โu!!ఐәдg & $Z / 9 t \angle 6$ & $\varepsilon \angle t 8 \angle \varepsilon$ & $6 \nrightarrow 0 \angle Z L G$ & $m$ & $m$ & & & & & & & & \\
\hline & $=88 Z \varepsilon \varsigma ८$ & ৪) & 凹 & 아 인 & ঠे: & ت & 8 & & q & $\stackrel{m}{-}$ & $=\sim$ & $\nabla 10 Z / L Z / G$ & 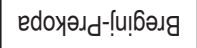 & $Z / 9+\angle 6$ & $90 \varepsilon 8 \angle \varepsilon$ & $090 \angle Z L G$ & $m$ & $m$ & & + & & & & & & \\
\hline & 06Z६९乙 & 응 & 凹 & ๑ & ঠ & $\Xi$ & ? & & প্ল & I & $\pm \infty$ & $\nabla 10 Z / L Z / G$ & 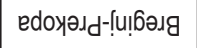 & $Z / 9 t \angle 6$ & $\varepsilon 0 \varepsilon 8 \angle \varepsilon$ & 9SOLZLG & + & + & & & & & & & & \\
\hline & $=$ Z6Z६૬乙 & চ্চি & $z$ & ? & 口: & ב & 8 & & m & m & $=\bar{N}$ & $\nabla 10 Z / / Z / 9$ & едоу્યәд-[u!!бәдg & $Z / 9 t \angle 6$ & $t 9 Z 8 \angle \varepsilon$ & ZLOLZLG & + & +- & $r$ & + & & & & & & \\
\hline & $68 Z \varepsilon \varsigma Z$ & 응 & 岕 & 인 & 口: & $\Xi$ & 8 & & 10 & $r$ & 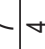 & $\nabla 10 Z / / Z / 9$ & едохудд-[u!!бәдg & $Z / 9+\angle 6$ & †0\&8LE & ISOLZLG & $\curvearrowright$ & $v+$ & & + & & & & & & \\
\hline & $96 Z \varepsilon \varsigma Z$ & ০০ & 㞱 & ஜ & 口: & ت & 8 & & 으 & $F$ & $=\approx$ & $\nabla\llcorner O Z / L Z / S$ & !ze|d-[u!!நəょg & $L / 9 t<6$ & Z69LLE & 9LLLZLG & - & - & & & + & . & & & & + \\
\hline & $00 \varepsilon \varepsilon \varsigma 乙$ & 员 & $\infty$ & 은 & 口: & ت & 8 & & 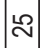 & $F$ & 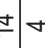 & $\nabla 10 Z / L Z / G$ & әуןәиәg-!u!దәдg & $l / 9 t<6$ & $8 \vdash t \angle L \varepsilon$ & $9 L \forall \angle Z L G$ & - & -+ & + & - & & & & & & \\
\hline & L6ZESZ & న్రి & $\infty$ & ৪) & 口: & $\Xi$ & 음 & & 으 & भ & $\sim \sim$ & $t レ O Z / L Z / S$ & !ze|d-[u!!ढə.g & $L / 9 t<6$ & G89LLE & EEZLZLG & - & -+ & + & & - & + & & & + & \\
\hline & ५৪Z६૬८ & న్రి & 岕 & ০ & 口: & $\Xi$ & 8 & & 와 & 용 & $=10$ & tレOZ/LZ/S & e|əg-[u!̣bəدg & $Z / 9+\angle 6$ & $99 t 8 \angle \varepsilon$ & 0t0LZLS & + & ++ & + & & & & & & & \\
\hline & \&৪Z६૬乙 & ০০ & $\infty$ & ৪) & 口: & $\Xi$ & 8 & & ৪) & 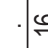 & $=$ & $\nabla レ O Z / L Z / S$ & е|әg-โu!|நәдg & $Z / 9 t \angle 6$ & LEऽ8LE & $900 \angle Z L G$ & + & +- & & & & & & & & \\
\hline & $\angle 8 Z \varepsilon \subseteq Z$ & চ্ & 岂 & 冓 & 口: & $\Xi$ & 8 & & 으 & m & $n \infty$ & $\nabla L O Z / L Z / S$ & edoyəıd-[u! & $Z / 9 t \angle 6$ & $00 \varepsilon 8 \angle \varepsilon$ & st0LZLS & + & +- & & + & & & & & & \\
\hline & 乙૦६६૬૪ & ০০ & 3 & 음 & 口: & $\Xi$ & 8 & & 요 & 이각 & $\pm ?$ & $t \downarrow O Z / L Z / S$ & 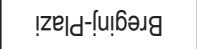 & $L / 9 t<6$ & I8LLLE & $9 \angle L \angle Z L S$ & 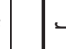 & - & - & & - & + & & & - & \\
\hline & IZ૬E९乙 & 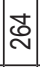 & $\sum_{z}^{3}$ & P & $\varangle$ & $\Xi$ & 8 & & 아: & Rন & $\sqrt[4]{2}$ & $\neq 10 z / \mathrm{L} / \mathrm{L}$ & ग्रəd e[zoy-ezin!pen & $t / 9 t<6$ & $97 \varepsilon \varepsilon 8 \varepsilon$ & 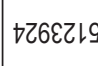 & - & - & & & & & & - & & \\
\hline & 乙IZE૬乙 & 导 & $z$ & ৪) & $\varangle$ & $\Xi$ & 8 & & 으 & $ㅇ$ & ما م م & tレOZ/OZ/9 & ग्रəd e[zoy-ezin!pen & $t / 9 t<6$ & $0 \varepsilon 6 Z 8 \varepsilon$ & st8\&ZLs & + & + & & & & & & & & \\
\hline & & & & & & & & 3 & $\overline{\mathbf{w}}$ & 이 & $\tilde{\varepsilon}$ & & & & $\varepsilon$ & $\varepsilon$ & i் & ㄷำ & 它 & $\bar{\omega}$ & $\bar{\Psi}$ & $\overleftarrow{\omega}$ & & 5 & $\overline{\mathbf{u}}$ & $\bar{\omega}$ \\
\hline & 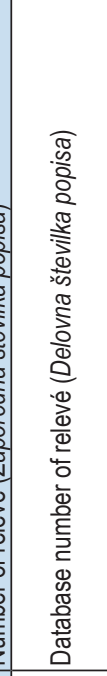 & 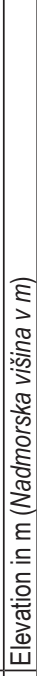 & & 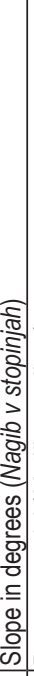 & 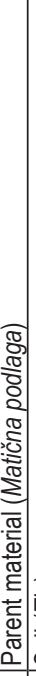 & $\underset{\mathbb{\sigma}}{=}$ & 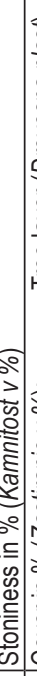 & 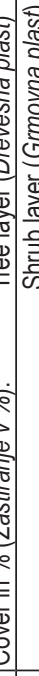 & 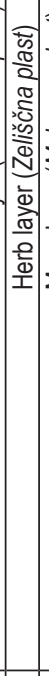 & 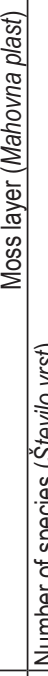 & 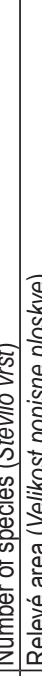 & 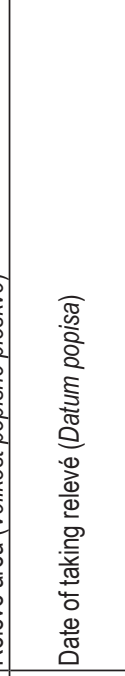 & 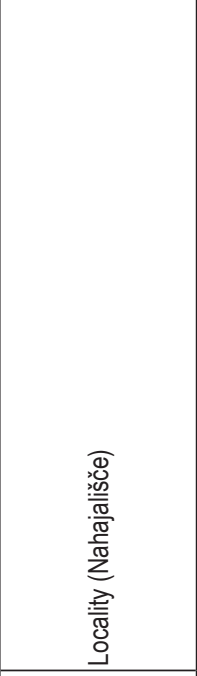 & 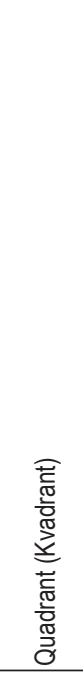 & 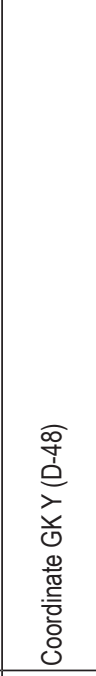 & 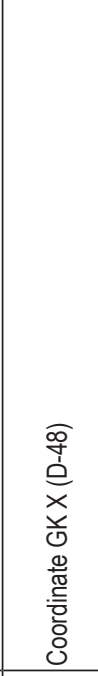 & 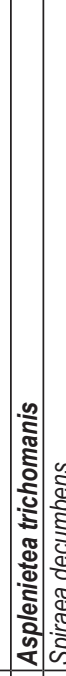 & 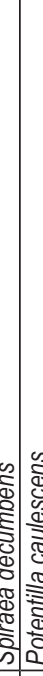 & 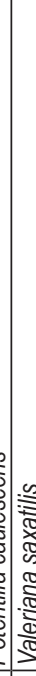 & 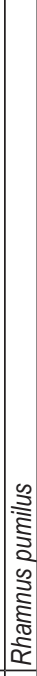 & & 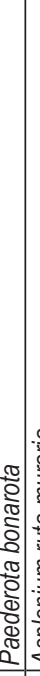 & & 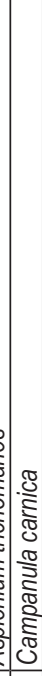 & 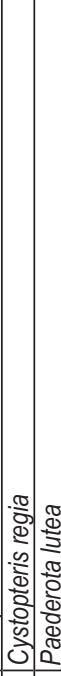 & 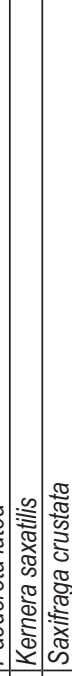 \\
\hline & & & & & & & & & & & & & & & & & 安 & & & & & & & & & \\
\hline
\end{tabular}




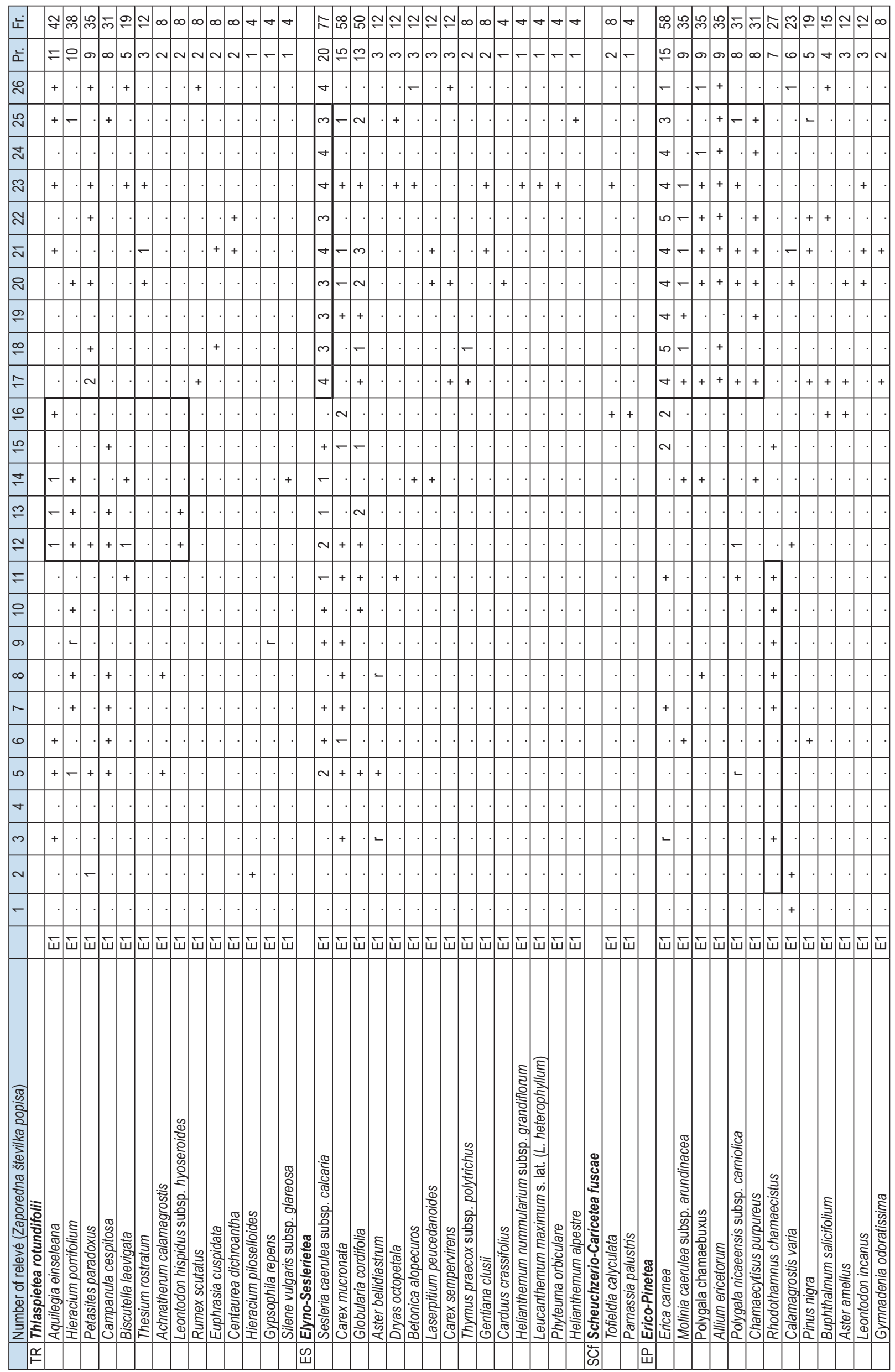




\begin{tabular}{|c|c|c|c|c|c|c|c|c|c|c|c|c|c|c|c|c|c|c|c|c|c|c|c|c|c|c|c|c|c|c|c|c|c|}
\hline ப் $\infty$ & $\infty \infty$ & $\infty \Rightarrow$ & $\checkmark \nabla$ & $\nabla+$ & & 9 & $\stackrel{\infty}{\stackrel{2}{2} \infty}$ & & $\infty \infty$ & + & & $+\nabla$ & $\nabla$ & $\cong \infty$ & $0 \infty \infty$ & & $\nabla \nabla$ & $\nabla$ & $\infty \nabla$ & & $\nabla$ & \% & $\approx \infty$ & & $9 \infty$ & o & + & & $+\Delta$ & $\nabla$ & $\nabla \nabla$ & $\checkmark \cong$ & m \\
\hline$\dot{\bar{\alpha}} \sim$ & $v \sim$ & & -- & -- & - & $\infty 0$ & $\nabla \sim$ & $\sim$ & $\sim \sim$ & - & $-r$ & -- & - & $m \sim$ & $v \sim \sim$ & $\sim$ & -- & - & $\sim$ & -5 & - & $\infty$ & $\sim \sim$ & & L 10 & $v-$ & -- & - & $-1-$ & - & - & $-m$ & 으 \\
\hline 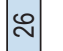 & & & & + & + & & + & & . + & & & & + & + & & + & & & & & + & & & & ++ & & & & & + & - & -- & - \\
\hline$\approx$ & & & & + & & & & & & & & & & & & & & & & & & + & & & & & & & & & & & \\
\hline A & & &.- & & & & & & & & & & & & & . & & & & & & & & & + & & & & & & + & & + \\
\hline N & & ++ & & & & . & - & ++ & ++ & & & & 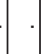 & + & ++ & & & & & & & & & & & & & & & & & & + \\
\hline$\approx$ & & & & & & & + & $\cdot+$ & + & & & & & &. & . & & & & & & & & & + & & & & & & & & + \\
\hline $\bar{N}$ & + & + & & & & + & & + & & & & & 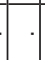 & + & +++ & + & & 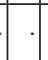 & + & & &. & $m$ & & & & & & & & & & + \\
\hline กิ+ & ++ & & & & & ++ & & & & & & + & & & & & & . & & & & 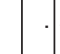 & - & & & & & & & & & & + \\
\hline ()+ & & & & & & & & & & & & & & + &.+ & & &. & & & & 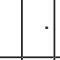 & + & & & & & & 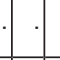 & & & & + \\
\hline$\infty$ & & & & & & + & & & & & & & & & & &. & + & & & & & & & & & & & . & & & & + \\
\hline$\neq$ & & & & & & + & -+ & & & + & + & - & & & . & & + & & + & & & & & & & & & & + & & & & + \\
\hline$\div$ & & & & & & & & & & & & & & & & & & & & & & - & & & . & & & & & & & & + \\
\hline$\stackrel{2}{\leftarrow}$ & & & & & & & & & & & & & & & & & & & & & & & & & & & & & & & & & \\
\hline 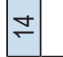 & & & & & & & & & & & & & & & & & & & & & & $\sim$ & & & + & & & & & & & - & \\
\hline$\stackrel{m}{=}$ & & & & & & + & & & & & & & & & & & & & & & & + & & & & & & & & & & + & \\
\hline$\cong$ & & & & & & & & & & & & & & & & & & & & & & 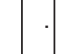 & + & & & & & & & & & & \\
\hline$\mp$ & & & & & & & & & & & & & & & & & & & & & & - & & & & & & & & & & & \\
\hline 으 & & & & & & & & & & & & & & & & & & & & & & & & & & & & & & & & & \\
\hline () & & & & & & & & & & & & & & & & & + & & & & & & & & & & & & & & & & \\
\hline$\infty$ & & & & & & & & & & & & & & - & - & & & & & & & & + & & & & & & & & & & \\
\hline$\sim$ & & & & & & & & & & & & & & & & & & & & & & & & & & & & & & & & & \\
\hline 0 & & & & & & & & & & & & & & & & & & & & & & & + & & & & & & & & & & \\
\hline 10 & & & & & & & & & & & & & & & & & & & & & & & ++ & & & & & & & & & & \\
\hline$\nabla$ & & & & & & & & & & & & & & & & & & & & & & & & & & & & & & & & & \\
\hline$m$ & & & & & & & & & & & & & & & & & & & & & & & & & & & & & & & & & \\
\hline & & & & & & & & & & & & & & & & & & & & -+ & & & & & - & + & + & - & & & & & \\
\hline & & & & & & & & & & & & & & & & & & & & & & & $\cdot+$ & & & & & & & & & & \\
\hline ш & $\bar{\Psi}$ & $\bar{\Psi}$ & $\bar{\Psi}$ & $\bar{\uplus}$ & & फั & $\bar{\omega}$ & फٓ & $\overline{\dot{\omega}}$ & $\bar{\omega}$ & $\bar{\Psi}$ & $\overline{\dot{\omega}}$ & i & $\overleftarrow{\dot{\omega}}$ & $\bar{ப} \overline{\dot{u}}$ & $\overline{\mathbf{u}}$ & $\bar{\Psi} \bar{\omega}$ & $\bar{\Psi}$ & $\bar{\omega}$ & $\bar{\Psi} \bar{\amalg}$ & $\bar{\omega}$ & జึำ & $\bar{\uplus}$ & & $\overline{\mathbf{u}}$ & $\bar{\nu}$ & $\bar{\Psi}$ & & & 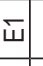 & & & \\
\hline
\end{tabular}

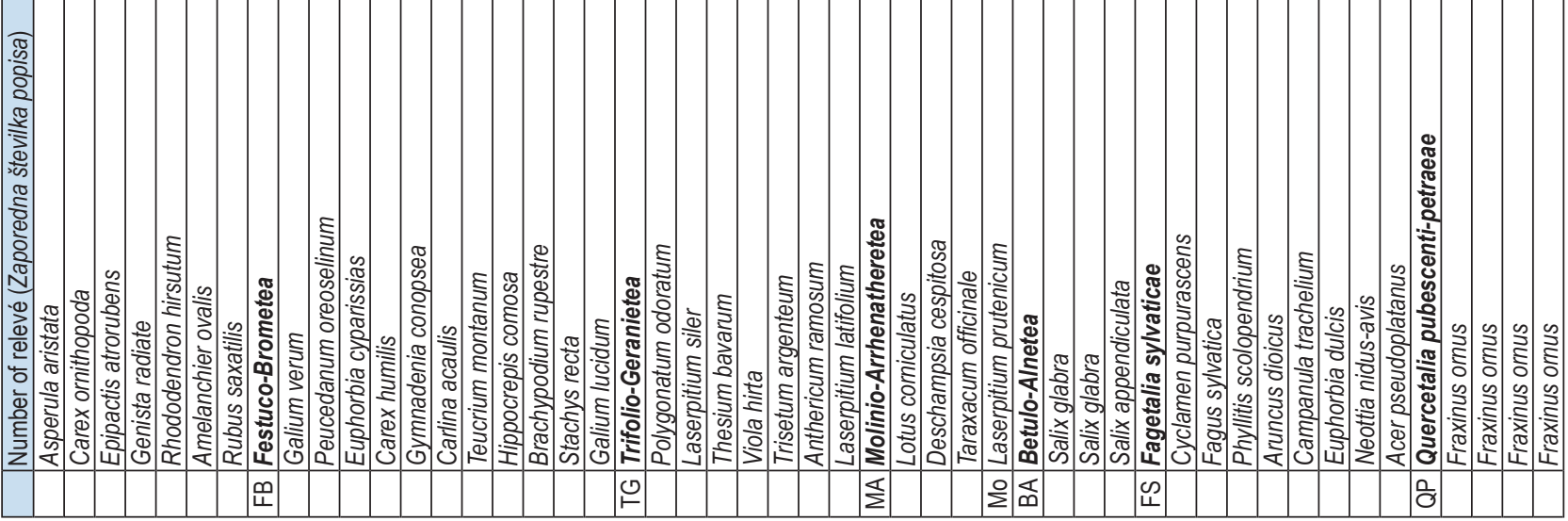



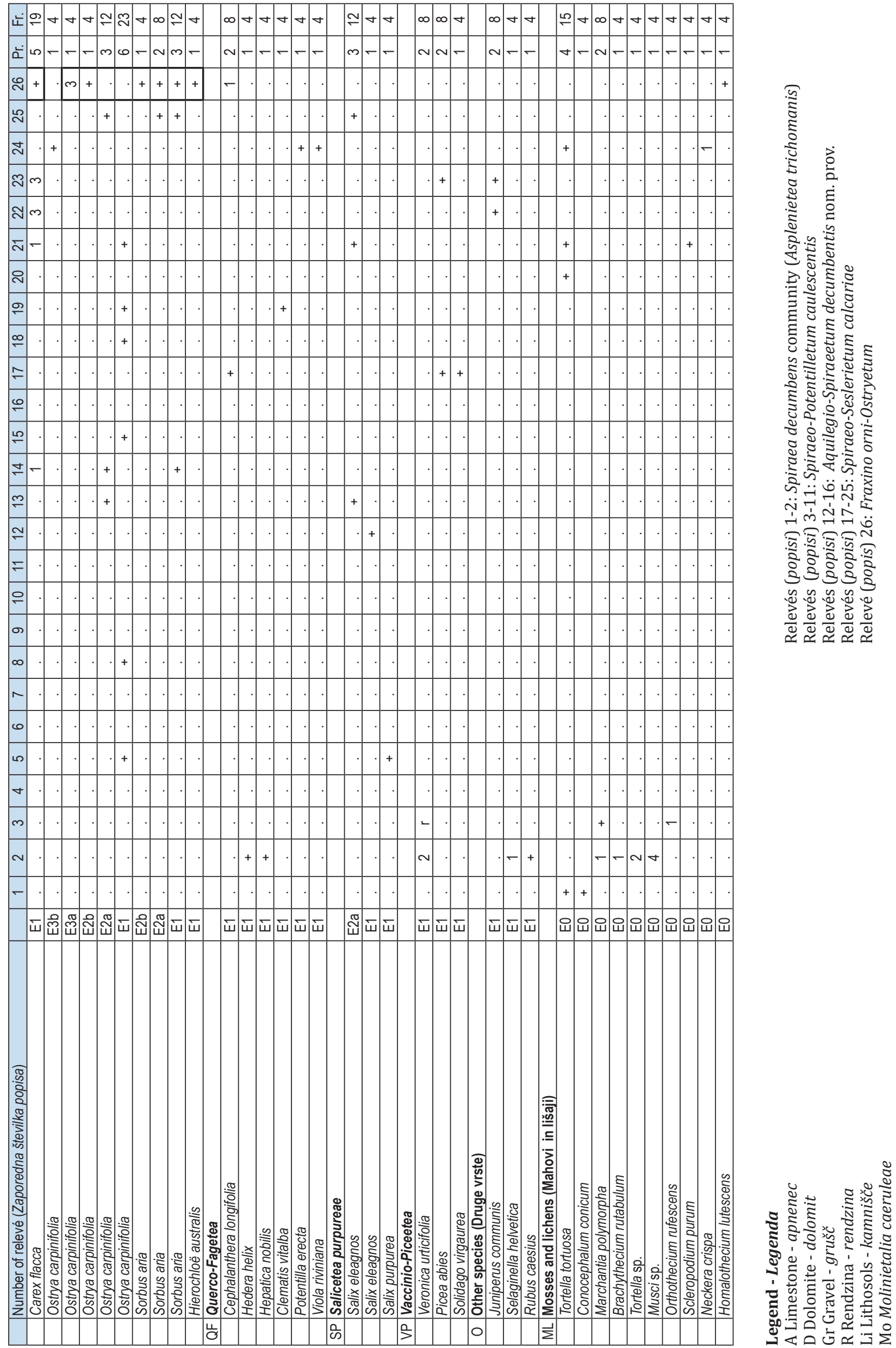
Table 2: List of areas with localities of Spiraea decumbens in Breginjski Kot
Preglednica 2: Seznam območij z nahajališči vrste Spiraea decumbens $\mathrm{v}$ Breginjskem kotu

\begin{tabular}{|l|c|c|c|}
\hline \multicolumn{1}{|c|}{ Area / Območje } & Number of relevés / Število popisov & Date of relevé /Datum popisa & Quadrant / Kvadrant \\
\hline Breginj-Bela & 9 & 21.5 .2014 & $9746 / 2$ \\
\hline Breginj-Prekopa & 6 & 21.5 .2014 & $9746 / 2$ \\
\hline Breginj-Plazi & 5 & 21.5 .2014 & $9746 / 1$ \\
\hline Breginj-Benetke & 3 & 21.5 .2014 & $9746 / 1$ \\
\hline Breginj-Klin & 1 & 21.5 .2014 & $9746 / 1$ \\
\hline Nadiža-Kozja peč & 2 & $11.7 .2014,20.6 .2014$ & $9746 / 4$ \\
\hline
\end{tabular}

\subsection{Overview of areas with localities of Spiraea} decumbens

3.2 Pregled območij z nahajališči vrste Spiraea decumbens

All determined localities of Spiraea decumbens were divided into six areas. Localities in the areas of Bela, Prekopa, Plazi, Benetke and Klin are shown in Figure 1, localities in the area of Kozja Peč in Figure 2.

\subsection{Description of Spireae decumbens populati- ons in Breginjski Kot}

3.3 Opis populacij vrste Spiraea decumbens v Breginjskem kotu

\subsubsection{Area Breginj-Bela}

\subsubsection{Območje Breginj-Bela}

This area comprises the river bed of a torrential tributary of the Bela, which is in the process of becoming overgrown. Its catchment area is under Mt. Musc. The overgrowing of screes began after 1933 when concrete torrential barriers were built there. This reduced flow velocity and prevented transportation of gravel. During the construction of barriers on the Bela, a large area was reforested, mainly with the conifers Picea abies, Pinus nigra, Pinus sylvestris, Larix decidua (Kočar, 1999). In this area, Spiraea decumbens grows between the last and its preceding barrier at the elevation between $695 \mathrm{~m}$ and $820 \mathrm{~m}$. Nine relevés were made there. The geological bedrock is talus or dolomite rock. Spiraea decumbens grows in two basic vegetation types, in stony grassland on overgrown scree (Spiraeo decumbentis-Seslerietum calcariae) and in a chasmophytic community (Spiraeo decumbentisPotentilletum caulescentis). Especially interesting is the secondary locality in the crevices on the uppermost concrete torrential barrier. Researchers have not reported such secondary localities for Slovenia until now, or they have not been known. Wraber (1969) saw them in Pušja Vas (Venzone) in Italy, where Spiraea decumbens grows on

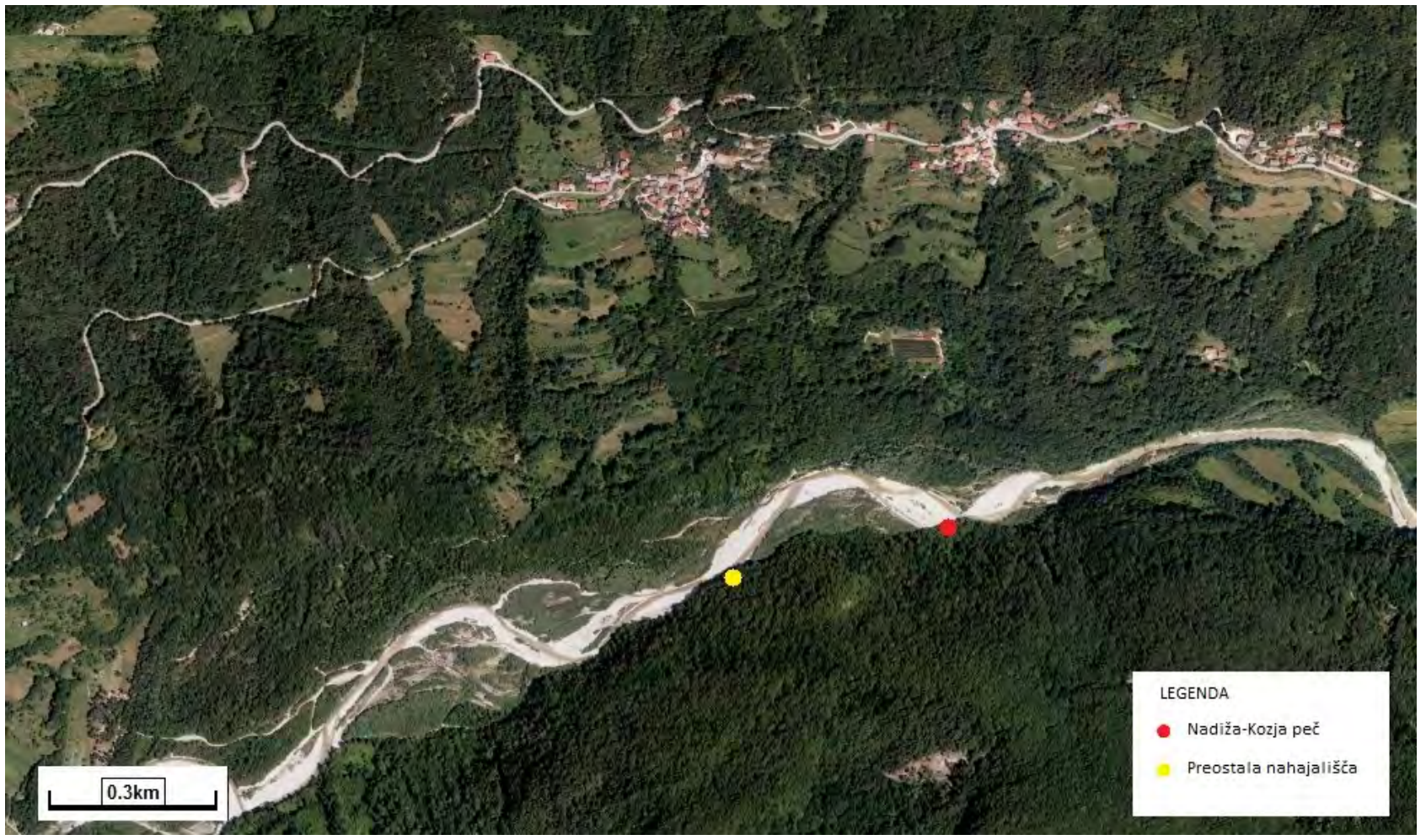

Fig. 2: Localities of Spiraea decumbens under Mt. Mija (Kozja Peč)
Slika 2: Nahajališča vrste Spiraea decumbens pod Mijo (Kozja peč) 
old walls. Most localities are on gentle slopes facing south and southeast; the slope ranges between $5^{\circ}$ and $35^{\circ}$, except on two localities where it reaches $80^{\circ}$ and $90^{\circ}$, respectively. The area occupied by Spiraea decumbens varies, ranging from $2.5 \mathrm{~m}^{2}$ to $12 \mathrm{~m}^{2}$ in size. On most of the plots it covers between $25 \%$ and $50 \%$ of the surface area (value 3 ), but it is very scarce on two plots where it covers only a small part of the surface area (value + ). The shrubs on stony grasslands grow to be the tallest, reaching up to 40 $\mathrm{cm}$ in height. Shrubs growing on the scree and in rock crevices are slightly shorter, up to $20 \mathrm{~cm}$. The population is vital and not potentially threatened.

\subsubsection{Area Breginj-Prekopa}

\subsubsection{Območje Breginj-Prekopa}

Prekopa is a botanically fascinating area with several habitats listed in Annex I to the Habitats Directive (Čušin, 2006). The slope gradient contributes to the erodibility of the dolomite slopes and the overgrowing of screes is very slow. Screes face towards the (south) east and (south)west and there is a mountain trail leading across the ridge towards Mt. Musc. All localities (six relevés) are on the top of the eastwards facing scree, but we did not find any localities on the western side. The elevation ranges between $900 \mathrm{~m}$ and $920 \mathrm{~m}$, the geological bedrock is talus or dolomite. Two vegetation types prevail: scree community (Aquilegio einseleanae-Spiraeetum decumbentis) and chasmophytic community (Spiraeo-Potentilletum caulescentis). Spiraea decumbens localities face north, northeast, east and southeast, on slopes of $40^{\circ}$ to $95^{\circ}$. The smallest sample plot measures $2 \mathrm{~m}^{2}$ and the largest $21 \mathrm{~m}^{2}$. Spiraea decumbens coverage varies among plots; it was assessed as + on three plots and as 1, 2 and 3 on the remaining three (according to the Braun-Blanquet scale, 1964). Shrub height does not exceed $20 \mathrm{~cm}$. The population is vital and not potentially threatened.

\subsubsection{Area Breginj-Plazi}

\subsubsection{Območje Breginj-Plazi}

Plazi is situated to the west of Prekopa. This area comprises known localities of several eastern-Alpine taxa that are rare in Slovenia: Thesium rostratum, Physoplexis comosa and Paederota bonarota. The first locality of Spiraea decumbens is only about a metre away from the road, on an already heavily overgrown slope planted with black pine. Other localities are situated at the bottom of two narrow gorges of the Plazi stream and on precipitous rock faces. It is in these two gorges that Spiraea decumbens population is the most abundant. The localities were recorded at $790 \mathrm{~m}$ to 820 m a.s.l., but Spiraea decumbens can grow even higher here, up to $1000 \mathrm{~m}$ a.s.l. The bedrock on four plots is dolomite and talus on one plot. Localities face different directions: west, northeast, south and east. Three plots, where we recorded a chasmophytic community, have a slope of $90^{\circ}$ to $100^{\circ}$ and two plots have a slope of $40^{\circ}$ or $50^{\circ}$. The smallest plot measures $2 \mathrm{~m}^{2}$ and the largest $12 \mathrm{~m}^{2}$. On most plots, Spiraea decumbens covers less than $10 \%$ of the surface area (value 1 ), whereas on one plot of $7 \mathrm{~m}^{2}$ it covers more than 50 $\%$ of the surface area. Shrub height ranges from $20 \mathrm{~cm}$ in rock crevices to $35 \mathrm{~cm}$ on scree. The Spiraea decumbens population on this locality is the largest, vital and not threatened.

\subsubsection{Area Breginj-Benetke}

\subsubsection{Območje Breginj-Benetke}

This is a steep dolomite erodible area of about 0.05 $\mathrm{km}^{2}$ to the west of Plazi. We made three relevés on spots that are not subject to erosion and allow for the growth of Spiraea decumbens. These localities are situated at an altitude between $820 \mathrm{~m}$ and $850 \mathrm{~m}$. Above the mountain pastures Ohoje and Javornik, localities can be found as high as $1200 \mathrm{~m}$. One plot is on slightly overgrown scree and two are in rock crevices. The localities face south, north and northwest. The slope on two plots is $40^{\circ}$ and $100^{\circ}$ on the third. The population of Spiraea decumbens is small here, with the largest plot measuring $4 \mathrm{~m}^{2}$ and the smallest only $1.5 \mathrm{~m}^{2}$. Spiraea decumbens covers more than $50 \%$ of the surface area on two plots and less than $10 \%$ on the third. Shrub height does not exceed $25 \mathrm{~cm}$. The population is vital and not threatened.

\subsubsection{Area Breginj-Klin}

\subsubsection{Območje Breginj-Klin}

The locality is situated on heavily overgrown scree, in an open forest stand on rendzina at about $100 \mathrm{~m}$ above the mountain trail leading to Brezje (Montemaggiore) village in Italy. The tree layer covers $70 \%$, the shrub layer $10 \%$ and the herb layer $80 \%$ of the plot. Only one locality nearby has a higher coverage, but could not be recorded because of the damages caused by sleet. The trees here are up to $6 \mathrm{~m}$ high and not thicker than $20 \mathrm{~cm}$. Thermophilous deciduous trees form the tree and the shrub layer: Fraxinus ornus, Ostrya carpinifolia and Sorbus aria. Fagus sylvatica and Acer pseudoplatanus occur in the herb layer along with manna ash and whitebeam. The geological bedrock consists of talus. The plot is situated $820 \mathrm{~m}$ a.s.l. and faces west. The slope gradient is $25^{\circ}$. Spiraea decumbens grows in an area of $20 \mathrm{~m}^{2}$ and covers less than $50 \%$ of the surface area. The tallest specimen of Spiraea decumbens 
was measured here at $50 \mathrm{~cm}$, whereas most shrubs on the plot reach the average height of $30 \mathrm{~cm}$. While vital, the population may become near threatened if the overgrowing of the scree continues.

\subsubsection{Area Nadiža-Kozja Peč \\ 3.3.6 Območje Nadiža-Kozja Peč}

At the foothills of Mija, under Kozja Peč on the right bank of the Nadiža, Marko Pavlin, the youngest of three authors, discovered on 20 June 2014 two new localities of Spiraea decumbens in the new quadrant 9746/4, about $400 \mathrm{~m}$ air distance apart. Igor Dakskobler picked a specimen for the Herbarium of the Institute of Biology SRC SASA (LJS) on 11 July 2014. These are for now the southeasternmost localities in the entire distribution area of this species and the first in the Mija Mountains. All other localities in the territory of Slovenia are in the Stol Mountains. There is only one shrub on each, which leads to the conclusion that the seeds were deposited by the Nadiža from its headwaters (hydrochory). The elevation of the plots was around $265 \mathrm{~m}$, the slope $70^{\circ}$ towards northwest (the first plot) and $90^{\circ}$ towards north (the second plot). In contrast to other 24 plots, the geological bedrock under Mija consists of limestone. The plot with easier access measures $1.5 \mathrm{~m}^{2}$ with Spiraea decumbens covering less than $10 \%$. The tallest specimens are below $30 \mathrm{~cm}$ and are vital. The river flow in this part is periodically regulated through gravel excavation and could become backfilled, so we alerted the Institute for Nature Conservation about this locality. The second locality under Kozja Peč is in much less accessible rocks above the Nadiža pool and could be threatened by visitors who come here for a summer swim. The distribution map of Spiraea decumbens in Slovenia with marked new locality is shown in Figure 3.

\section{CONCLUSIONS}

\section{ZAKLJUČKI}

We recorded 47 localities of the southeastern-Alpine endemic species Spiraea decumbens in five areas (the Bela headwaters, Prekopa, Plazi, Benetke and Klin) under the Stol ridge in Breginjski Kot (northwestern Slovenia) in the elevation belt of $700 \mathrm{~m}$ to $1200 \mathrm{~m}$. All of them are situated in the existing quadrants of the Central-European flora mapping grid, 9746/1 and 9746/2. Based on the relevés made on 21 localities, we classified the recorded stands into the following associations: Spiraeo decumbentis-Potentilletum caulescentis Poldini 1969, Aquilegio einseleanae-Spiraeetum decumbentis nom. prov., Spiraeo decumbentis-Seslerietum calcariae Dakskobler in Pavlin, Brus et Dakskobler 2015 ass. nov. and Fraxino orni-Ostryetum Aichinger 1933 corr. Franz 2002. Both, the provisionally and newly described association are successional stages in the overgrowing of dolomite screes and torrential fans with thermophilous deciduous trees, in particular Ostrya carpinifolia, Fraxi-

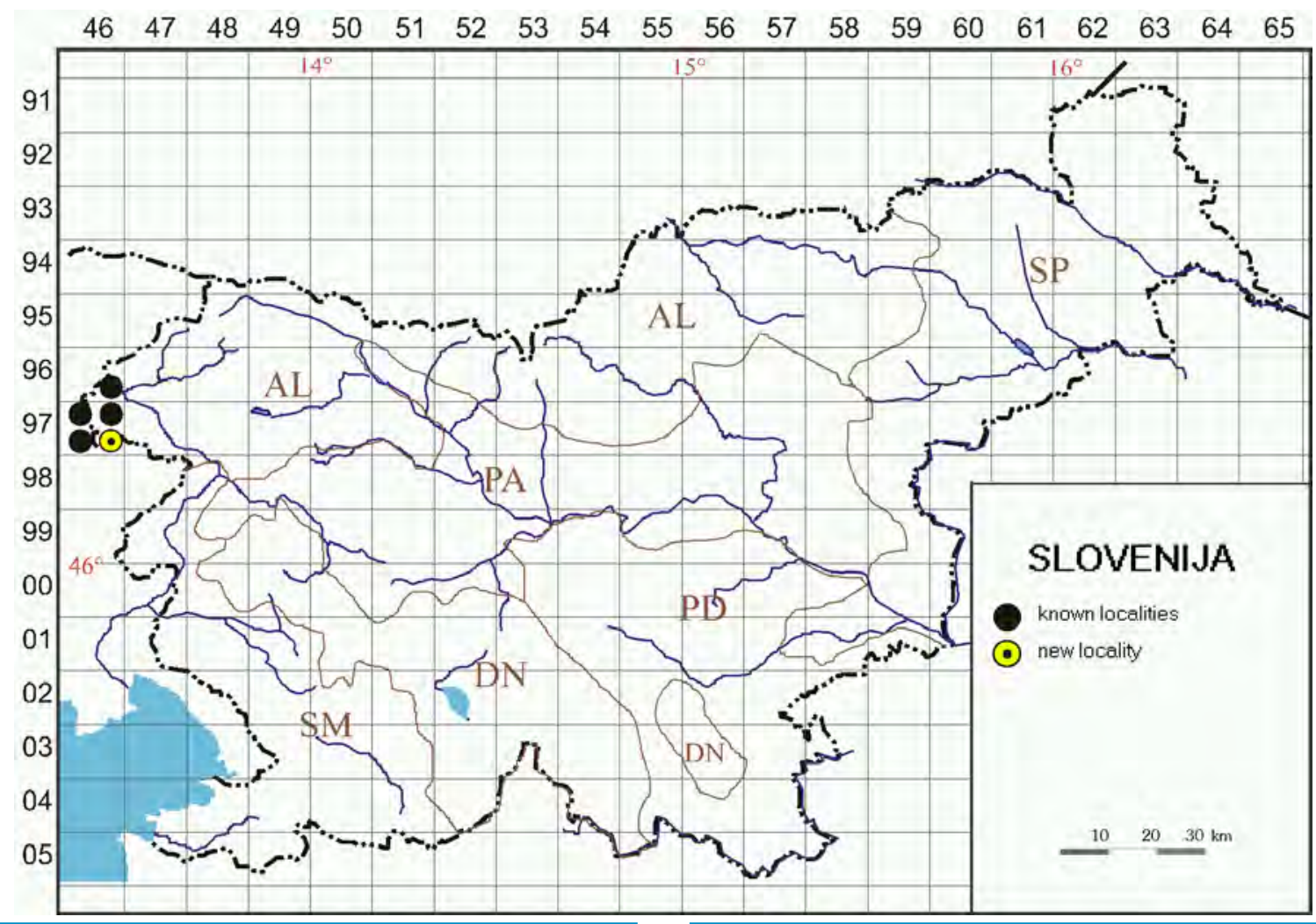

Fig. 3: Distribution of Spiraea decumbens s. str. in Slovenia 
nus ornus and Sorbus aria, sporadically also with individual Pinus nigra trees. Despite several attempts, we did not manage to confirm the Spiraea decumbens locality at the gravel bar on the Nadiža that had been reported by Wraber (1969) and which Čušin (2006) had failed to find as well. However, we found two new localities in the new quadrant (9746/4) at the northern foot of Mt. Mija, at Kozja Peč on the right bank of the Nadiža River. These two localities differ from all other localities investigated in our study, both in terms of ecology (the geological bedrock is limestone) and floristics. Only two clusters of Spiraea decumbens overgrow steep riverine rocks and overhangs. We assume that its seeds had been deposited here by water. Spiraea decumbens is potentially threatened by swimmers in the new localities under Mija and even more by gravel extraction that takes place with the regulation of the river bed. This small population should therefore be carefully monitored and protected, especially because it is the southeasternmost locality in its entire distribution area. On other localities, Spiraea decumbens thrives either in small patches or tufts, or in large clusters in areas ranging from $1.5 \mathrm{~m}^{2}$ to $24 \mathrm{~m}^{2}$ in size. The largest population of Spiraea decumbens was found in the area Plazi, where favourable site conditions facilitate its growth in a larger area (screes, rock barriers and solitary rocks). The vitality of individuals on most localities is good, even on slightly shaded sites. Dry individuals that have probably died off because of their physiological age are only rarely observed. The species also regenerates well, although only in limited areas. All this points to predominantly vegetative propagation, which is most distinct in rock crevices.

\section{POVZETEK}

\section{SUMMARY}

Na petih območjih (povirje Bele, Prekopa, Plazi, Benetke in Klin) pod Stolovim grebenom v Breginjskem kotu (severozahodni Slovenija) smo popisali 47 nahajališč jugovzhodnoalpskega endemita polegle medvejke (Spiraea decumbens) v višinskem pasu od okoli $700 \mathrm{~m}$ do $1200 \mathrm{~m}$. Vsa so v že znanih kvadrantih srednjeevropskega kartiranja flore, 9746/1 in 9746/2. Na 21 nahajališčih smo naredili tudi fitocenološki popis in popisane sestoje uvrščamo v naslednje asociacije: Spiraeo decumbentis-Potentilletum caulescentis Poldini 1969, Aquilegio einseleanae-Spiraeetum decumbentis nom. prov., Spiraeo decumbentis-Seslerietum calcariae Dakskobler in Pavlin, Brus et Dakskobler 2015 ass. nov. in Fraxino orni-Ostryetum Aichinger 1933 corr. Franz 2002. Obe, provizorna in novo opisana asociacija, sta sukcesijska stadija v zaraščanju dolomitnih melišč in hudourniških vršajev s tolploljubnimi listavci, pred- vsem črnim gabrom, malim jesenom in mokovcem, posamično tudi s črnim borom. Kljub večkratnemu iskanju nam ni uspelo potrditi nahajališča polegle medvejke na prodišču pri Mostu na Nadiži, ki ga navaja Wraber (1969) in ga je zaman iskal tudi Čušin (2006). Našli pa smo dve novi nahajališči v novem kvadrantu (9746/4) na severnem vznožju Mije, pri Kozji peči na desnem bregu Nadiže. Ti dve nahajališči se tako ekološko (geološka podlaga je apnenec) kot floristično precej razlikujeta od vseh drugih pregledanih nahajališč. Vrsta Spiraea decumbens v dveh šopih porašča strme do previsne obrečne skale in je njeno seme najbrž prinesla voda. Na obeh novih nahajališčih pod Mijo poleglo medvejko potencialno ogrožajo kopalci in predvsem izkopavanje proda med regulacijami rečne struge, zato mora biti ta mala populacija deležna skrbnega spremljanja in varstva, tudi zato, ker je najbolj jugovzhodna v celotnem arealu. Na drugih nahajališčih polegla medvejka raste $\mathrm{v}$ majhnih krpah oziroma blazinicah ali večjih šopih na površini od $1,5 \mathrm{~m}^{2}$ do $24 \mathrm{~m}^{2}$. Njeno največjo populacijo smo ugotovili v območju Plazi. Tam so zanjo na večji površini ugodne rastiščne razmere (melišča, skalni pragovi in osamelci). Vitalnost osebkov je na večini nahajališč dobra, tudi na že nekoliko zasenčenih rastiščih. Le redko lahko ponekod opazimo suhe osebke, ki so najverjetneje odmrli zaradi fiziološke starosti. Vrsta se tudi dobro pomlajuje, vendar le na omejenih površinah. Iz tega sklepamo na prevladujoče vegetativno razmnoževanje. Najbolj je to opazno v skalnih razpokah.

\section{ACKNOWLEDGEMENTS}

\section{ZAHVALA}

The study is the result of the graduation thesis (Pavlin, 2015) written by the younger author under the mentorship of the senior authors. Sincere thanks to Prof. Dr. Franc Batič for his insights and comments. Dr. Lado Kutnar and Dr. Aleksander Marinšek helped us with valuable improvements and corrections. English translation by Andreja Šalamon Verbič.

\section{REFERENCES}

\section{LITERATURA}

Aeschimann D., Lauber K., Moser D. M., Theurillat J.-P. 2004. Flora alpina. Bd. 1: Lycopodiaceae-Apiaceae. Bern, Stuttgart, Wien,Haupt Verlag: 1159 p.

Anonymus 2002. Pravilnik o uvrstitvi ogroženih rastlinskih in živalskih vrst v rdeči seznam. Priloga 1: Rdeči seznam praprotnic in semenk (Pteridophyta \& Spermatophyta). Ljubljana, Uradni list RS 82/2002.

Braun-Blanquet J. 1964. Pflanzensoziologie. Grundzüge der Vegetationskunde. 3.izd. Wien; New York, Springer: 865 p.

Čušin B. 2006. Rastlinstvo Breginjskega kota. Ljubljana, Založba ZRC, ZRC SAZU: 198 p. 
Dakskobler I. 2003. Floristične novosti iz Posočja in sosednjih območij v zahodni Sloveniji - III. Hladnikia (Ljubljana), 15-16: 43-71. Geopedia. Interaktivni spletni atlas in zemljevid Slovenije. http:// www.geopedia.si/lite.jsp\#T105_x384232_y123488_s13_b4

Jogan J. 2000. Razširjenost medvejk (Spiraea spp.) v Sloveniji. Annales, Ser. hist. nat., Koper, 10, 1: 81-90.

Kočar T. 1999. Nekdanji Breginj: Bela, mlini, žaga, gozdovi in drugo. Breginj: Turistično društvo: $123 \mathrm{p}$.

Martinčič A. 2003. Seznam listnatih mahov (Bryopsida) Slovenije. Hacquetia (Ljubljana), 2, 1: 91-166.

Martinčič A., Wraber T., Jogan N., Podobnik A., Turk B., Vreš B., Ravnik V., Frajman B., Strgulc Krajšek S., Trčak B., Bačič T., Fischer M. A., Eler K., Surina B. 2007. Mala flora Slovenije. Ključ za določanje praprotnic in semenk. Četrta, dopolnjena in spremenjena izdaja. Tehniška založba Slovenije, Ljubljana: 967 p.
Martinčič A. 2011. Seznam jetrenjakov (Marchantiophyta) in rogovnjakov (Anthocerotophyta) Slovenije. Scopolia (Ljubljana), 72: 1-38.

Pavlin M. 2015. Razširjenost rodu medvejka (Spiraea L.) v Breginjskem kotu. Diplomsko delo. Univerza v Ljubljani, Biotehniška fakulteta, Oddelek za gozdarstvo in obnovljive gozdne vire, Ljubljana: 33 p.+ Appendix.

Podani J. 2001. SYN-TAX 2000. Computer Programs for Data Analysis in Ecology and Systematics. User's Manual, Budapest: 53 p.

Poldini L. 1973. Lo Spiraeo-Potentilletum caulescentis associazione rupicola delle Alpi Carniche. Atti Mus. civ. St. nat. Trieste, 28, 2: 451-463.

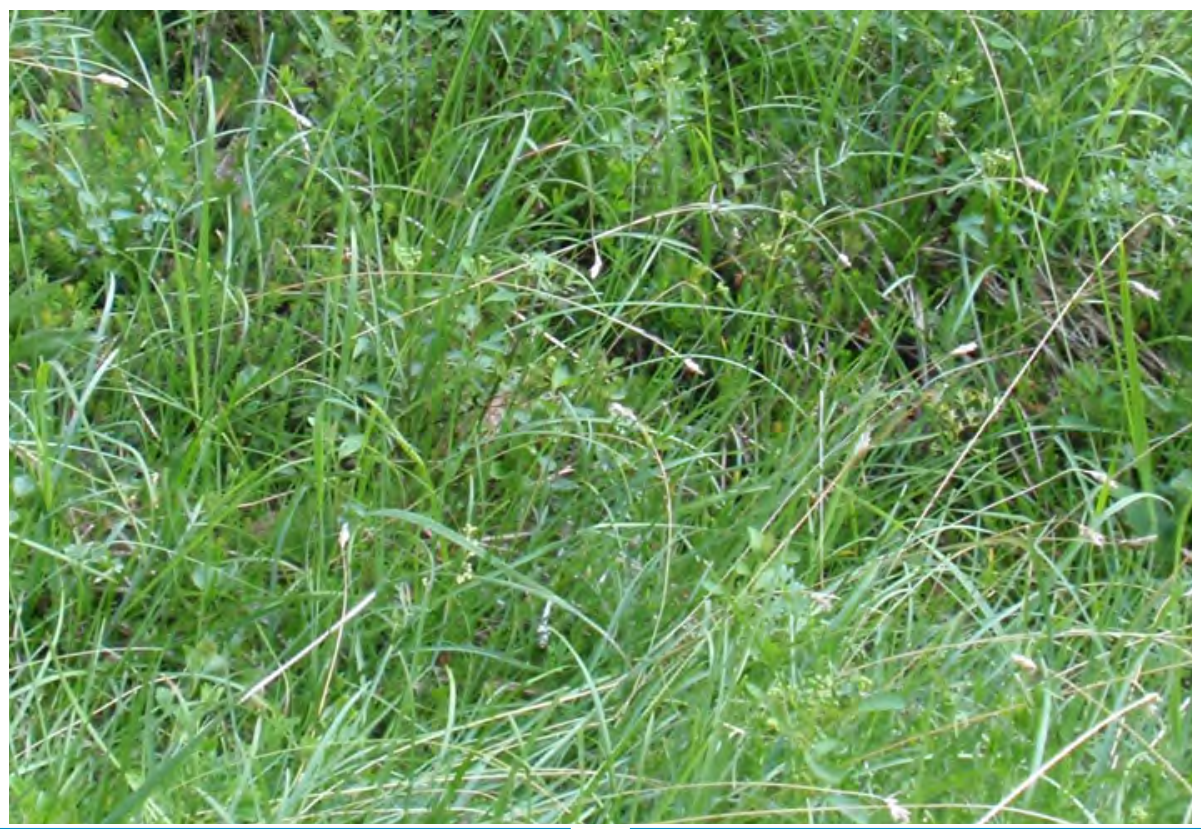

Fig. 4: Stand of the association Spiraeo decumbentis-Seslerietum calcariae. Photo: M. Pavlin
Slika 4: Sestoj asociacije Spiraeo decumbentis-Seslerietum calcariae. Foto: M. Pavlin

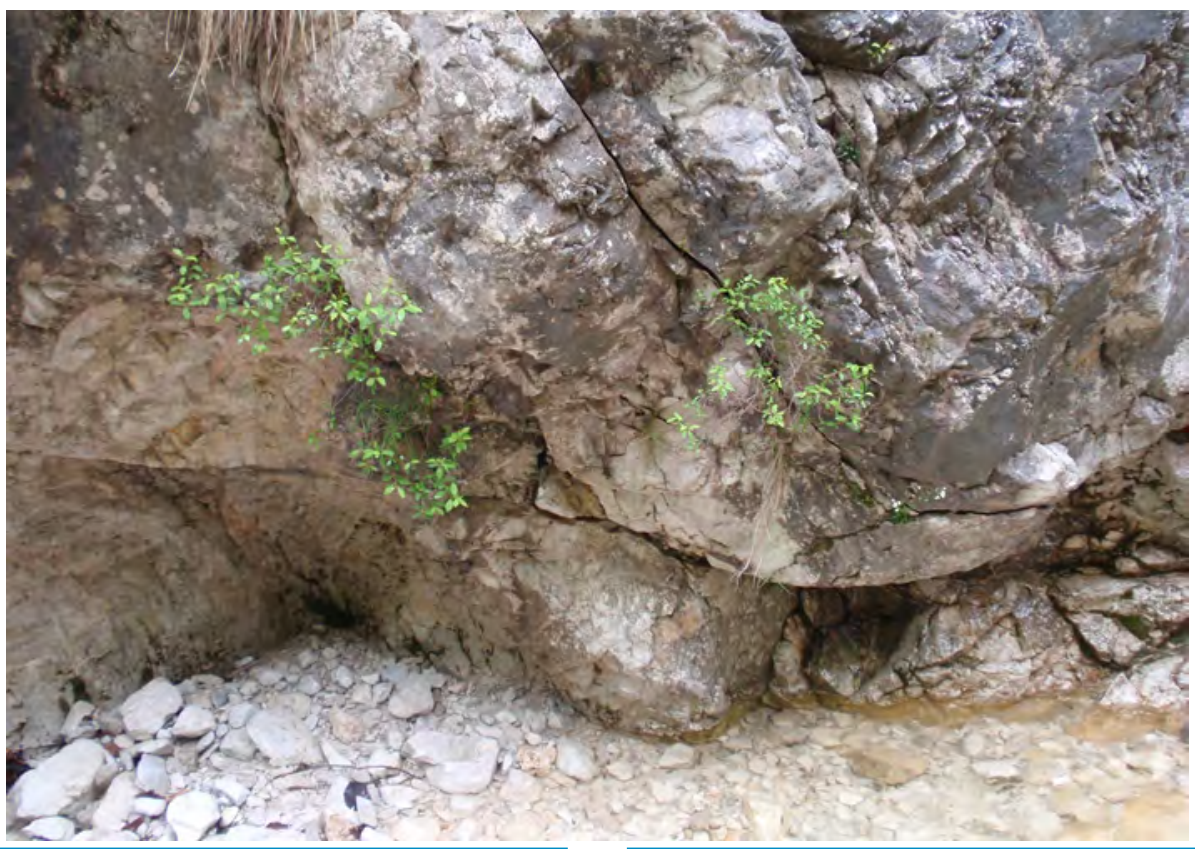

Fig. 5: Stand of the association Spiraeo decumbentis-Potentilletum caulescentis. Photo: M. Pavlin
Slika 5: Sestoj asociacije Spiraeo decumbentis-Potentilletum caulescentis. Foto: M. Pavlin 
Poldini L. (with collaboration of G. Oriolo \& M. Vidali), 2002. Nuovo Atlante corologico delle piante vascolari nel Friuli Venezia Giulia. Udine,Regione Autonoma Friuli Venezia Giulia, Azienda Parchi e Foreste Regionali \& Universitá degli Studi di Trieste, Dipartimento di Biologia: $529 \mathrm{p}$.

Seliškar T., Vreš B., Seliškar A. 2003. FloVegSi 2.0. Računalniški program za urejanje in analizo bioloških podatkov. Biološki inštitut ZRC SAZU, Ljubljana.

Šilc U., Čarni A. 2012. Conspectus of vegetation sintaxa in Slovenia. Hacquetia (Ljubljana), 11, 1: 113-164. van der Maarel E. 1979. Transfomation of cover-abundance values in phytosociology and its effects on community similarity. Vegetatio (Den Haag), 39, 2: 97-114.

Wraber T. 1969. Nekatere nove ali redke vrste v flori Julijskih Alp (III). Varstvo narave (Ljubljana), 6: 73-84.

Wraber T., Skoberne P. 1989. Rdeči seznam ogroženih praprotnic in semenk SR Slovenije. Varstvo narave (Ljubljana), 14-15: 1-429.

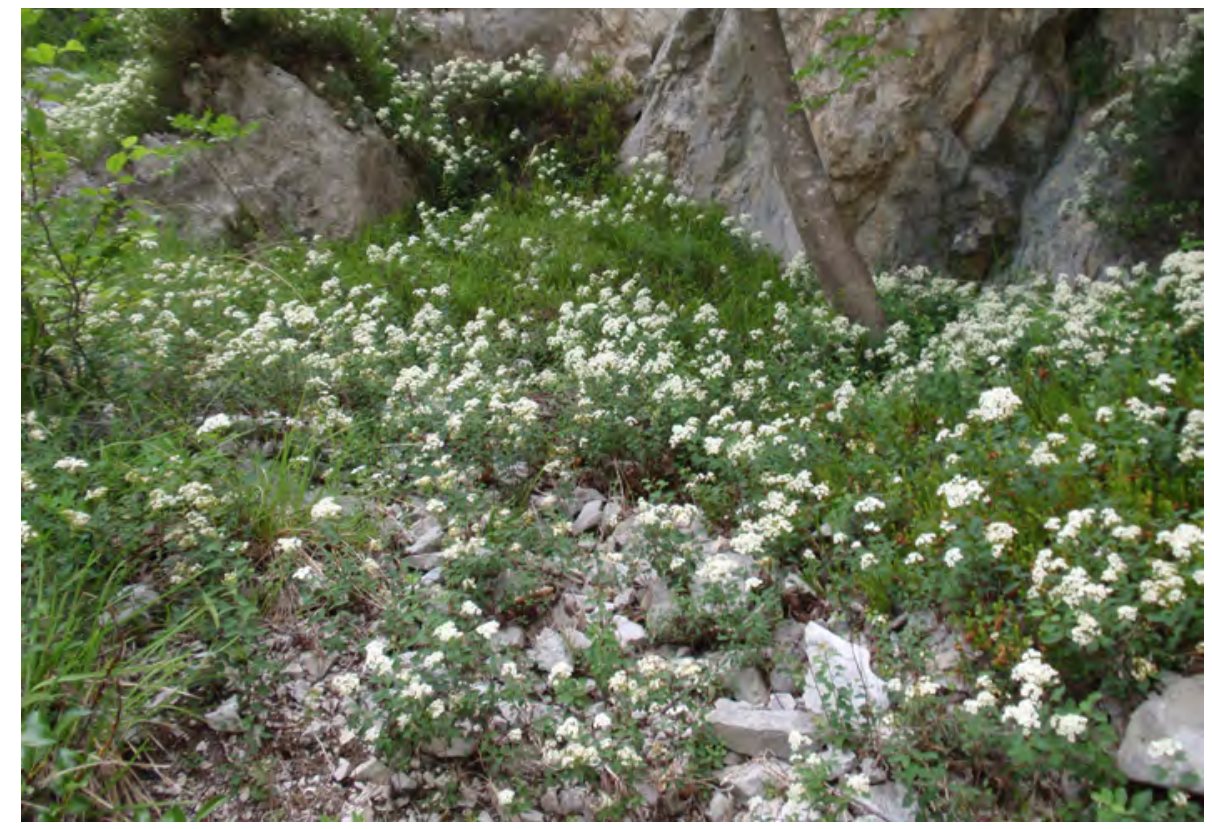

Fig. 6: Stand of the provisional association Aquilegio einseleanae-Spiraeetum decumbentis. Photo: M. Pavlin
Slika 6: Sestoj provizorne asociacije Aquilegio einseleanaeSpiraeetum decumbentis. Foto: M. Pavlin

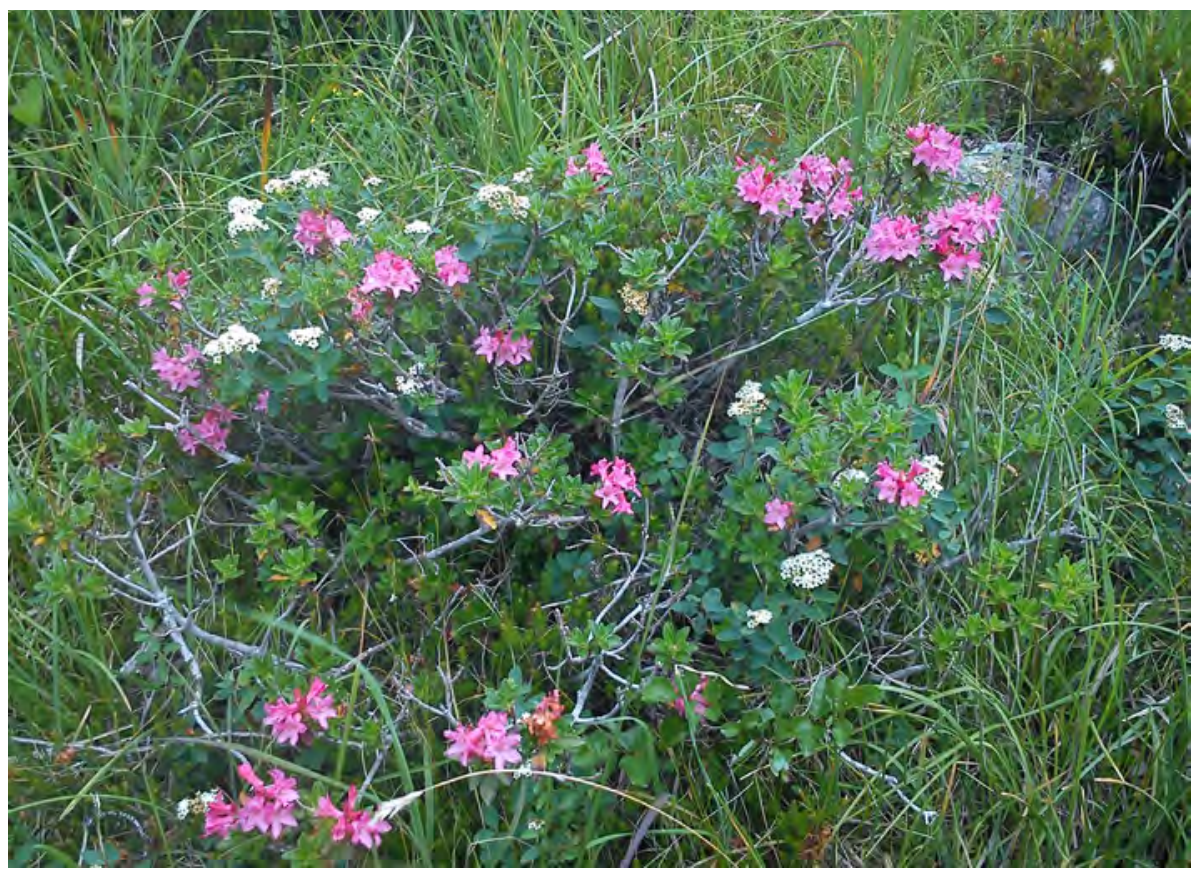

Fig. 7: Spiraea decumbens and Rhododendron hirsutum flourishing on overgrown scree in the stand of the association Spiraeo decumbentis-Seslerietum calcariae. Photo: M. Pavlin
Slika 7: Polegla medvejka in dlakavi sleč cvetita v sestoju asociacije Spiraeo decumbentis-Seslerietum calcariae. Foto: M. Pavlin 


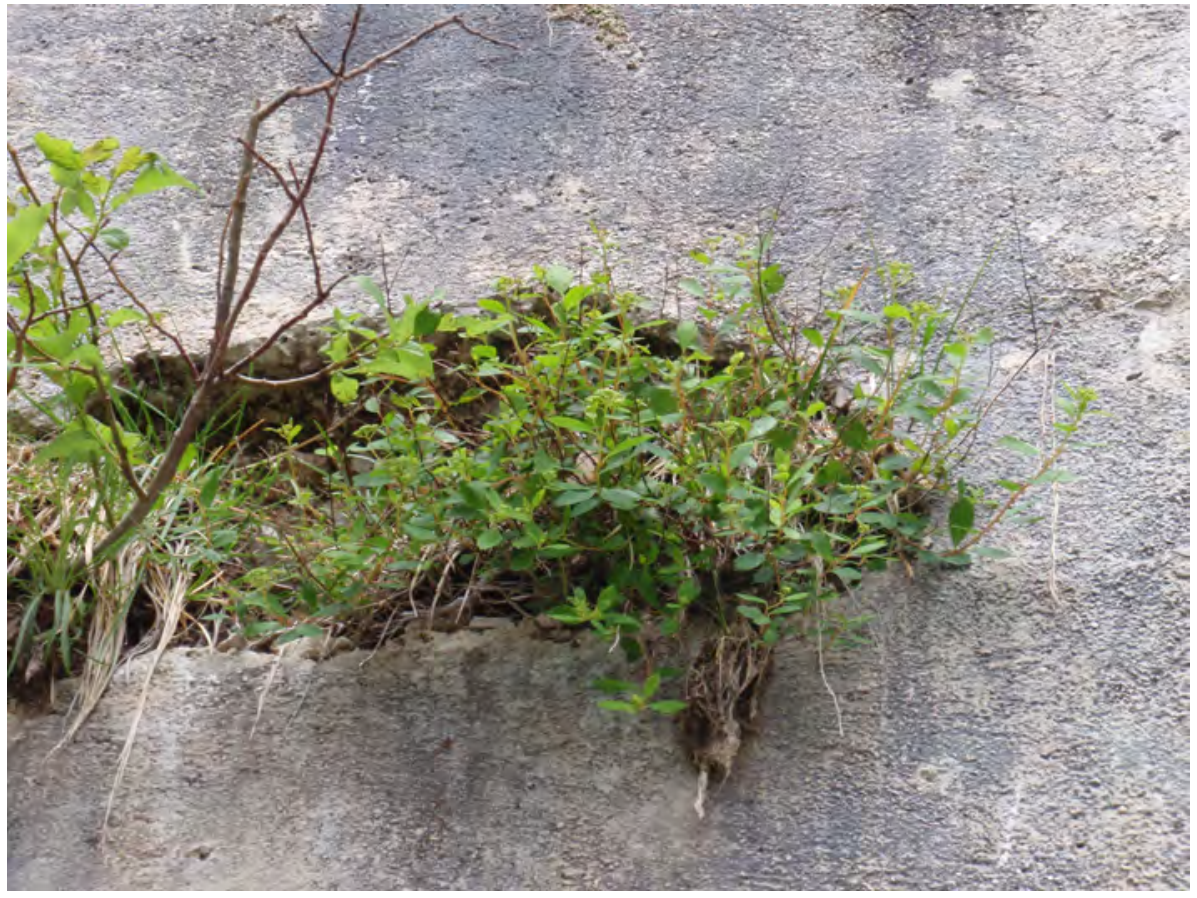

Fig. 8: Secondary locality of Spiraea decumbens in the crevices on the uppermost concrete torrential barrier in the channel of a torrential tributary of the Bela. Photo: M. Pavlin

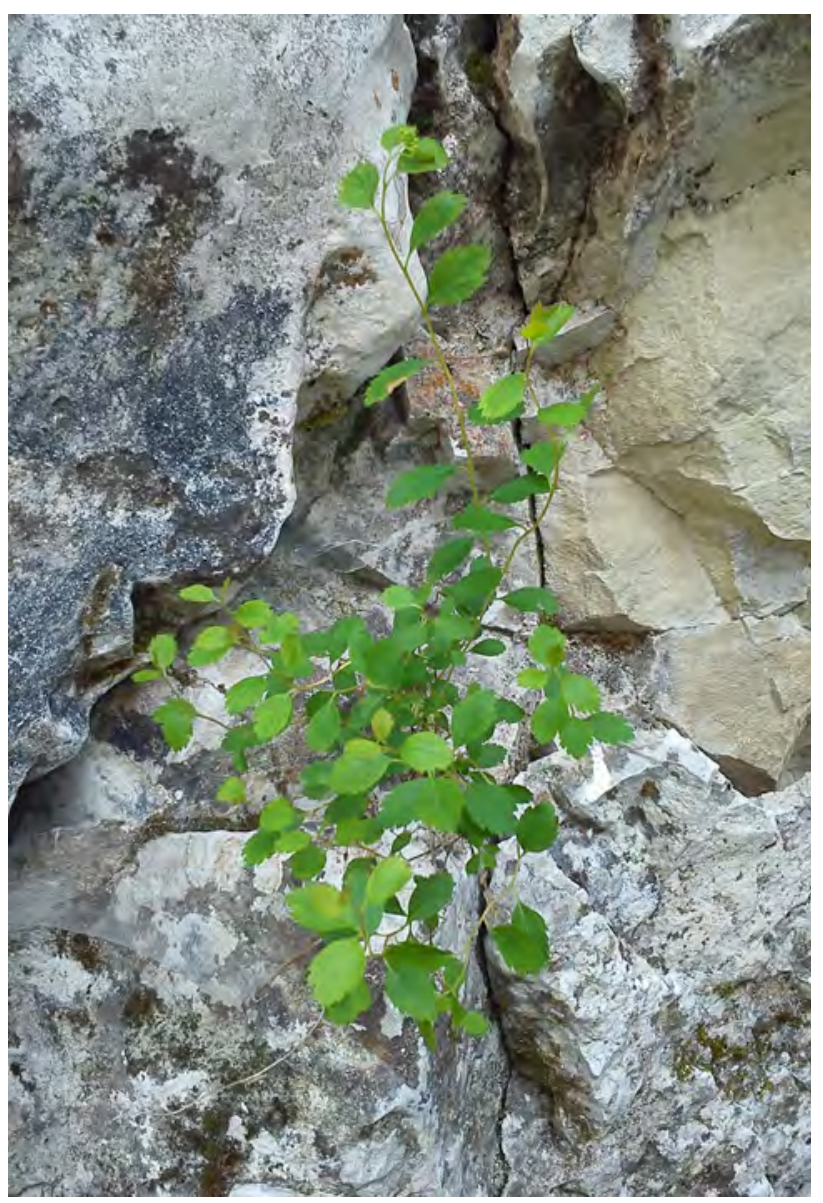

Fig. 9: New locality of Spiraea decumbens near Kozja Peč on the right bank of the Nadiža River. Photo: M. Pavlin

Slika 9: Novo nahajališče polegle medvejke pri Kozji peči na desnem bregu Nadiže. Foto: M. Pavlin
Slika 8: Subspontano pojavljanje polegle medvejke na betonski pregradi v hudourniški strugi Bele. Foto. M. Pavlin

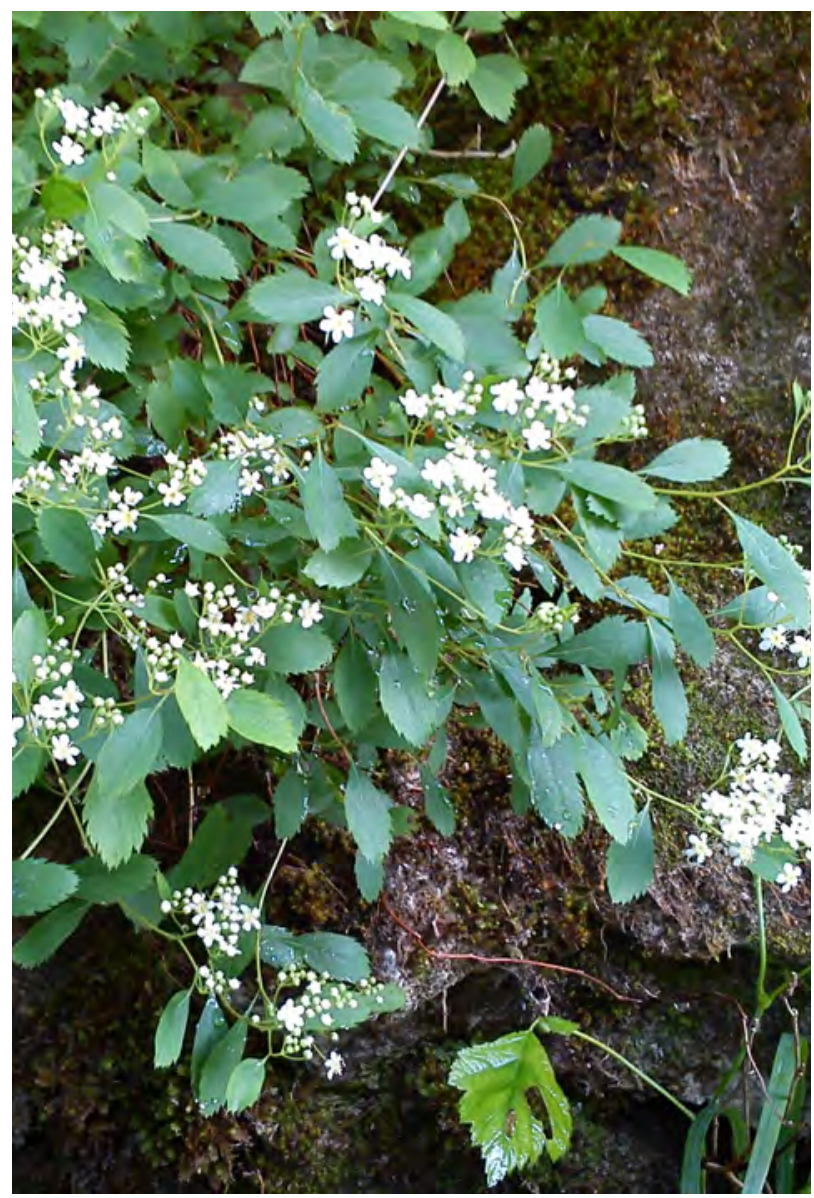

Fig. 10: The second new locality of Spiraea decumbens near Kozja Peč on the right bank of the Nadiža. Photo: M. Pavlin Slika 10: Drugo novo nahajališče polegle medvejke pri Kozji peči na desnem bregu Nadiže. Foto: M. Pavlin 